\title{
Autour de la sculpture et du tactile : jeux de langage(s), conduites d'élèves et arts de faire professionnels en cycle 1
}

"Yes, you can touch the sculptures!" Pre-elementary students exploring a tactile exhibition: langage games, forms of museum life, and professional skills

Françoise Arbouet, Danielle Bertrand, Marie-Do Bidard, Jean-Charles Chabanne, Manon Gaquerel, Philippe Mesmin, Mary-Ėve Penancier, Stéphanie Rieu et Cécile Salvador

\section{(2) OpenEdition}

Édition électronique

URL : http://journals.openedition.org/pratiques/3678

DOI : $10.4000 /$ pratiques.3678

ISSN : 2425-2042

Éditeur

Centre de recherche sur les médiations (CREM)

Référence électronique

Françoise Arbouet, Danielle Bertrand, Marie-Do Bidard, Jean-Charles Chabanne, Manon Gaquerel, Philippe Mesmin, Mary-Ėve Penancier, Stéphanie Rieu et Cécile Salvador, « Autour de la sculpture et du tactile : jeux de langage(s), conduites d'élèves et arts de faire professionnels en cycle $1 »$, Pratiques [En ligne], 175-176 | 2017, mis en ligne le 22 décembre 2017, consulté le 19 avril 2019. URL : http:// journals.openedition.org/pratiques/3678 ; DOI : 10.4000/pratiques.3678

Ce document a été généré automatiquement le 19 avril 2019.

(c) Tous droits réservés 


\section{Autour de la sculpture et du tactile : jeux de langage(s), conduites d'élèves et arts de faire professionnels en cycle 1}

"Yes, you can touch the sculptures!" Pre-elementary students exploring a tactile exhibition: langage games, forms of museum life, and professional skills

Françoise Arbouet, Danielle Bertrand, Marie-Do Bidard, Jean-Charles Chabanne, Manon Gaquerel, Philippe Mesmin, Mary-Ėve Penancier, Stéphanie Rieu et Cécile Salvador

\section{Introduction}

1 Comment établir un lien dynamique, non réducteur, entre ce qui se joue dans la rencontre avec des œuvres d'art dans un lieu d'exposition, et les apprentissages scolaires ? Comment préparer, conduire et prolonger de tels moments pour les enrichir et les densifier (sans les dénaturer ou les réduire par une conception réductrice du « scolaire »), ce qui fait la valeur de l'évènement que constitue, pour une classe, une visite dans cet autre lieu, qu'est un musée?

Dans cet article, ces questions sont posées en lien avec des préoccupations de formation initiale et continue des enseignants, qui se déclarent souvent démunis par ce qu'ils perçoivent comme des expériences riches, qu'ils ne voudraient pas réduire au récréatif. En effet, ils ont conscience des liens évidents avec des contenus importants comme le langage, la culture, la capacité à questionner, la mise en place d'une familiarité avec les pratiques culturelles, l'initiation à une approche à la fois sensible et réfléchie aux pratiques artistiques, toutes finalités qui sont inscrites à la fois dans les programmes scolaires au titre de l'éducation artistique et culturelle (MEN, 2015a, 2015b, 2015c) et dans 
une politique coordonnée des ministères de l'Éducation et de la Culture, constamment réaffirmée depuis plusieurs décennies (MEN\&MCC, 2015 ; MCC, 2017).

Les visites aux musées sont des réalisations concrètes de ce projet de politique éducative conjoint. Quelles questions identifient les acteurs dans cette mise en œuvre à échelle réelle? Comment y répondre avec eux? Nous présentons ici, de manière cursive, les éléments de réponse que le LéA Musécole ${ }^{1}$ a commencé à élaborer et expérimenter, à la recherche de situations considérées comme emblématiques.

\section{Contexte, acteurs, questions}

\section{Une recherche collaborative}

4 Le collectif Musécole est inscrit dans le réseau des lieux d'éducation associés (LéA), initié par l'Institut français de l'éducation (IFÉ), dans une certaine conception de la « recherche collaborative orientée vers la conception " (Sanchez \& Monod-Ansaldi, 2015). Celle-ci se définit comme un réseau d'échanges courts entre les fonctions pratique, recherche, formation, pilotage. La trentaine de LéA en activité forment un laboratoire-observatoire de tels projets (Chabanne, Monod-Ansaldi, \& Loisy, 2016²). Le LéA Musécole cherche ainsi à rapprocher sans les confondre les missions de documentation, de questionnement et de validation qui sont celles de la recherche ; les missions de conception de dispositifs ou de ressources qui sont celles de la formation, à partir des questionnements qui remontent des praticiens dans les contextes concrets des classes et des institutions éducatives; enfin, il tient compte des commandes institutionnelles et de la prescription.

C'est cette préoccupation qui réunit, depuis plusieurs années, un groupe de rechercheformation qui associe des personnels chargés de l'accueil du public dans un musée, des enseignants-formateurs et des formateurs-chercheurs d'une école supérieure du professorat et de l'éducation (ESPÉ). Le groupe répond à une double demande : du côté de l'institution muséale, le besoin de donner aux enseignants de quoi préparer, conduire et exploiter des visites pour les très jeunes publics (maternelle); du côté de l'institution de formation, le besoin de former à l'éducation artistique et culturelle (ÉAC), désormais part importante de la commande politique. L'ambition est que les enseignants puissent donner dans leur programmation plus de temps à ce type d'activités dans un espace continu entre école et musée, pour tisser ensemble la fonction médiation-métier et la fonction enseignement. Au rebours de la division du travail que la séparation du scolaire et du hors scolaire voudrait établir, nous pensons au contraire que chaque espace professionnel, celui des métiers de l'accueil des visiteurs in situ, et celui des métiers de l'enseignement, ont en commun une responsabilité sociale (l'ÉAC), mais aussi des gestes professionnels, en particulier celui de la médiation-action.

\section{Définir médiation et éducation culturelle}

6 La perspective de la formation nous amène à nous intéresser aux compétences professionnelles sollicitées par ce que nous appellerons médiation-action: accompagner sur place (mais aussi préparer et prolonger) les évènements que sont les rencontres avec ces objets particuliers qu'on appelle des œuvres d'art. Dans notre article, le terme médiation-action désignera l'activité d'étayage de la rencontre matérielle avec l'œuvre, in situ. Ce travail est, dans nos observations, accompli tantôt par les enseignants eux- 
mêmes, tantôt par un.e professionnel.le du service des publics. Ainsi le terme est dangereusement polysémique: dans la terminologie des métiers du musée et de l'exposition, la médiation est désormais une spécialité professionnelle (Mörsch \& Chrusciel, 2012; Lamizet, 1999). L'intérêt est qu'il se déploie dans des définitions beaucoup plus vastes: dans une problématique anthropologique (Caune, $1999 ; 2017)$, ou pour la sociologie de l'art (Heinich, 2009). À défaut d'une meilleure option, et pour garder au terme son épaisseur, nous lui donnons ici un sens restreint, distinguant la médiation(action) de la médiation-métier et le médiateur(-acteur) du médiateur professionnel.

7 La médiation ainsi définie est étroitement liée à la dimension culturelle évoquée dans l'expression Éducation artistique et culturelle, elle vise un effet éducatif. De nombreux travaux ont porté et portent encore sur la possibilité d'évaluer les effets spécifiques de l'ÉAC sur une vaste palette d'apprentissages (Bamford, 2008; Winner, Goldstein, \& Vincent-Lancrin, 2014 ; Bordeaux \& Kerlan, 2015). Ces « apprentissages » comportent une dimension instrumentale (on y apprend par exemple certaines techniques pour observer et comparer, un certain vocabulaire descriptif, des notions d'histoire, etc.). Mais ils engagent aussi des dimensions plus vastes, des processus de socialisation profonds, qui les inscrivent dans le projet éducatif global (on y apprend par exemple certains usages du corps, certaines manières de parler; à s'initier à des manières de sentir, de penser, de représenter, qui nous sont étrangères ou distantes, etc.; on y apprend à être attentif, tolérant, critique, etc. ; on y apprend à réfléchir, mais aussi à (res)sentir, à interpréter, à discuter, à mettre en lien...) (Barton, 2013).

\section{Une attention particulière portée à la question du (et des) langage(s)}

Reste à justifier la présence de cette contribution dans une revue à priori spécialisée en didactique du français. D'abord, il s'agit de répondre à l'invitation stimulante lancée par Pratiques à un échange pluridisciplinaire sur la problématique de la «médiation». Or, l'ÉAC inclut pleinement les arts du langage, la littérature, pour laquelle se posent les mêmes questions : comment articuler processus de médiation de l'expérience sensible de l'œuvre et visées d'apprentissage ou d'éducation? Dès 1995, un colloque organisé par Pratiques s'interrogeait sur les effets d'une scolarisation de la lecture (Beaude, Petitjean, \& Privat, 1996), dans la période où l'on se demandait s'il ne fallait pas «déscolariser la lecture » (Foucambert et al., 1982).

9 Mais en outre, la didactique du français est définie par son intérêt pour « la réception et la production des discours oraux et écrits» (Halté, 2008). Elle ne peut donc qu'être intéressée par les pratiques langagières et sémiotiques qui s'entrelacent aux pratiques de médiation-action. Elle y retrouve son intérêt pour les médiations sémiotiques de l'expérience de lecture (Rouxel \& Langlade, 2004 ; Doquet, Lumbroso \& Tauveron, 2009 ; Ahr \& Joole, 2013). Ces questions ne sont pas nouvelles dans son champ : elles prolongent directement des questions esthétiques très anciennes, comme celle de l'ekphrasis (Webb, 1999), le dialogue entre les arts (Vouilloux, 1997), la problématique des écrits sur l'art (Vaugeois, 2005). L'apport des spécialistes de la parole sur l'art et de ses genres (y compris le discours de l'histoire de l'art et de la critique) éclaire l'ÉAC selon une perspective particulière.

10 Enfin, une grande part de la compétence du médiateur-acteur consiste à savoir produire et interpréter de multiples jeux de signes, ces jeux de langage qui, dans les situations observées, constituent à la fois le moyen par lequel les connaissances sont présentées et 
partagées, mais aussi, de manière moins visible, le moyen par lequel est lancée, accompagnée et pensée l'expérience sensible. Le langage verbal (mais aussi d'autres langages, nous le rappellerons) est pour les élèves et leurs guides un ensemble d'outils pour rendre possible puis garder en mémoire l'évènement de la rencontre.

\section{Un contexte : l'exposition L'Art et la matière au musée Fabre, une galerie de sculptures à toucher (printemps 2017)}

11 Le LéA Musécole travaille en fonction de l'actualité du contexte de travail. Dans les deux dernières années, le musée Fabre, partenaire du LéA, a préparé la mise en place d'une galerie tactile, qui permet au public de toucher des sculptures. Dans le prolongement d'autres expériences, à l'origine liées à l'accueil des non-voyants, c'était aussi l'occasion d'une expérimentation de ce que peut apporter l'expérience tactile à l'expérience sensible, visuelle, et à l'approche intellectuelle (Kastrup \& Sampaio, 2012; Lemoine \& Simier, 2016).

L'exposition se présente dans une salle dont l'aménagement scénographique permet à des visiteurs de tous âges d'avoir à portée de la main des copies d'œuvres, la plupart au format original, de dimensions variées : statues en pied, groupes animaliers, bas-relief, statue équestre en réduction ${ }^{3}$... Le public est accueilli par un panneau d'initiation à l'exploration tactile (boites à toucher, échantillons de matériaux...). Un autre espace expose quelques outils de modelage, l'éventail des techniques de la sculpture et un film sur le moulage au bronze.

\section{La méthode de travail : à la recherche de gestes professionnels et de situations emblématiques}

13 Les données sont collectées pendant les visites : des enregistrements vidéo, des travaux d'élèves comme les dessins réalisés in situ. Sont aussi documentées les séances qui préparent et prolongent la visite, par exemple des ateliers préparant aux problématiques de la sculpture, des séances de restitution à partir de photographies, etc. Autoconfrontations, analyses croisées, entretiens, notes de travail forment un autre ensemble de matériaux. Le LéA travaille ainsi simultanément sur des questions relevant des contenus éducatifs potentiels (qu'est-ce qui est supposé s'apprendre là ?), et sur les logiques professionnelles des intervenants.

14 La perspective de l'outil de formation est présente dès cette étape : comment saisir et rendre accessible ces éléments de savoir-faire? C'est le troisième niveau de travail du LéA, la production de ressources, dont l'expérimentation auprès de divers publics renvoie à d'autres questions sur les enjeux, les conduites d'élèves, les gestes professionnels, dans un processus de conception continuée dans l'usage (Sanchez \& Monod-Ansaldi, 2015).

La préoccupation initiale du LéA est de permettre à des enseignants en formation de travailler sur un matériau qui permette de s'interroger à la fois sur les enjeux de savoir, les conduites d'élèves et certains gestes professionnels que nous appelons emblématiques. 


\section{Définition de " geste professionnel »}

16 La notion de "geste professionnel », aujourd'hui très utilisée, a été définie de manière assez différente par les auteurs qui s'y sont attachés (Jorro, 2006; Bucheton \& Soulé, 2009 ; Bernié \& Goigoux, 2005 ; Alin, 2010). Nous lui donnons une définition pragmatique et exploratoire : pour nous, les gestes professionnels sont ce qui préoccupe en priorité un groupe de professionnels échangeant sur ce qu'ils ont à faire dans un certain type de situation. Ils sont ainsi définis comme des unités élémentaires de ce qui serait la compétence professionnelle. Ils se révèlent ainsi tout autant dans des moments difficiles, que dans des moments de réussite, de satisfaction, d'étonnement positif.

\section{Encadré 1. Accompagner l'expérience de l'œuvre : identifier un art de faire}

Classe de Philippe, petite et moyenne section. Les élèves, dans la première phase de la séance, entrent dans la galerie les yeux bandés, et sont amenés vers la première sculpture (La Frileuse) par les mamans accompagnatrices. Dans un premier temps, ils tâtonnent, tournent autour de l'œuvre, hésitent, et visiblement leurs gestes deviennent plus erratiques. Ils s'en éloignent et commencent à jouer entre eux. Visiblement, leur attention décroit très vite, et l'effet de surprise passée ils semblent ne plus savoir quoi faire. Les mamans se contentent de rappeler la consigne : il faut toucher... mais elles non plus ne savent pas trop ce qui est en-jeu $u^{4}$.

Arrive Philippe : avec quelques mots il les fait revenir vers la sculpture : le tâtonnement devient plus méthodique, et surtout un dialogue s'installe, où les enfants prennent la parole en parallèle avec des gestes plus méthodiques. Nos questions commencent ici : qu'est-ce qui était présupposé et qui ne se produit pas dans la première phase? Que leur a dit l'adulte, qui capte leur attention et lance à la fois une verbalisation et une exploration tactile visiblement coordonnées? Que sait le médiateur-acteur qui lui permet d'intervenir, quelles sont ses règles d'action, dont on peut constater qu'elles ne sont pas improvisées puisqu'il les reproduira à d'autres moments, devant d'autres œuvres - et qu'il sera capable, dans les analyses, de les restituer, de les organiser, et de les partager avec d'autres?

Comment passer de cette description intuitive à une formalisation minimale qui permette de concevoir un outil léger de formation? Par exemple : prendre conscience de l'intérêt didactique et pédagogique de telle consigne ; pointer la pertinence et la variété des enchainements sur les propositions des élèves ; etc. On voit que ces décisions professionnelles impliquent une réflexion sur ce que signifie faire usage du toucher pour apprendre à voir et à sentir : la situation, dans sa simplicité, appelle les questions de fond.

On voit comment le LéA envisage ainsi de construire une ressource pour la formation : non pas lister de manière abrupte et décontextualisée des consignes figées, mais proposer de quoi observer et questionner ce moment fugace, non pas pour le considérer comme un modèle, mais comme une source de réflexion non modélisante, emblème d'une question laissée ouverte. 


\section{Présentation de quelques gestes professionnels identifiés}

17 On voit la difficulté de l'entreprise engagée, puisqu'il s'agit d'établir une liste de gestes tout en considérant qu'elle doit rester mobile et provisoire, qu'elle doit guider sans modéliser. Le travail ainsi défini est en cours, et devra être ajusté par des expérimentations en formation, auprès de divers publics. Nous présentons dans ce qui suit un travail encore en cours, sur des éléments partiels qui forment à ce stade une matrice provisoire.

\section{Identifier, sélectionner et traduire les connaissances mises en jeu : présenter le savoir, prendre la parole au juste moment}

Nous commencerons par la première des préoccupations que formulent les étudiants, et ce n'est pas la plus simple : leur première objection est qu'ils «n'y connaissent rien » et qu'ils ne savent pas "quoi dire " aux élèves. Ce qui se joue ici est effectivement une question fondamentale : qu'est-il supposé se passer au cours de la visite, et en particulier qu'est-il supposé « s'apprendre » au travers de cette expérience, qui ne pourrait s'enseigner ailleurs?

On peut tout de suite objecter qu'il peut y avoir d'autres en-jeux que la seule capacité à restituer des savoirs verbalisables, des noms propres, des dates, des catégories esthétiques, des noms de matériaux, des définitions, des récits, des explications, des descriptions, etc. On verra plus loin qu'on peut interroger cette première définition. Mais prenons-la pour commencer.

Le travail collaboratif dans le LéA fait d'ailleurs apparaitre que cette question est aussi partagée par les médiateurs, y compris justement pour refuser d'en faire un en-jeu principal : les connaissances sont utiles, mais elles doivent venir à leur moment et à leur juste mesure - quand et sous quelle forme et jusqu'à quel point de détail faut-il apporter du savoir, voilà justement sinon un geste professionnel, du moins un vrai problème. Ainsi, l'analyse de la scène autour de La Frileuse montre que les consignes du médiateur sont guidées par ce qu'il veut que les élèves apprennent et comprennent, qu'il a donc, lui, une idée de ce qui est en-jeu à ce moment précis de la visite.

Cela renvoie à un questionnement beaucoup plus large, et qui concerne le projet même de cette galerie, pas seulement auprès d'un public scolaire. Au fond, pour tout visiteur, qu'est-ce qui peut s'y passer, qu'est-ce qui peut s'y expérimenter, qu'est-ce qui peut s'y apprendre? Ce questionnement est d'abord celui des commissaires d'exposition, et il relève de toutes les sciences de l'art : histoire, technologie, sémiologie, psychologie... comme le montrent les conférences qui accompagnent l'exposition (cycle Fabre Lab, mars 20175). Ce sont des questions de fond: Qu'est-ce qu'une «sculpture »? Pourquoi les a-t-on créées? Quand les a-t-on fabriquées, selon quelles techniques ? Où les trouve-t-on, comment sont-elles mises en scène dans les lieux où elles étaient exposées? Quels ont été leurs usages, leurs fonctions et leurs significations? Plus largement, que disait et que dit toujours la sculpture, en quoi les questions qu'elle pose trouvent toujours écho dans nos sociétés? Poser ces questions, y répondre, en discuter, c'est une grande partie de ce que les politiques éducatives appellent une "culture artistique» (voir par exemple les 
attendus du programme d'histoire des arts [MEN, 2008] ou la définition du parcours d'ÉAC [MCC, 2017]). Ce premier ensemble de connaissances semble impressionnant : mais il n'est pas autre chose que l'arrière fond théorique qu'appelle la nouvelle discipline scolaire, posée comme transversale à tout le programme de l'École : l'Histoire des arts (Chabanne, $2013 ; 2017)$.

\section{Le choix d'un lexique}

Un premier geste professionnel relève d'un travail de transposition ou si l'on veut, suivant U. Eco (2006), de traduction. Choisir, adapter et mettre à disposition les connaissances, cela se manifeste centralement par le choix d'une terminologie, et par le problème éminemment didactique posé par la formulation du texte du savoir (Chevallard, 1991): cartels, affichages, brochures; commentaires des audioguides ou improvisés in situ. On peut aussi le définir par ce que peut en restituer le visiteur.

Groupe Petite section. Devant Enfant jouant avec une levrette ${ }^{6}$, Manon demande qu'on nomme la matière dans laquelle l'œuvre a été sculptée. Elle nous dira qu'elle cherche à établir une liste de matériaux avec eux.

Tableau 1. Extrait du verbatim

\begin{tabular}{|l|l|}
\hline M & le sol, c'est froid, c'est dur \\
\hline E1 & ma tête aussi // et c'est blanc \\
\hline E2 & le chien aussi ici, c'est blanc \\
\hline M (pointant vers chaque détail) & $\begin{array}{l}\text { les balcons en quoi ils sont les balcons, la } \\
\text { balustrade, là ? }\end{array}$ \\
\hline Eointant la porte) & là derrière vous c'est en quoi ? \\
\hline M & alors c'est en bois \\
\hline E1 & $\begin{array}{l}\text { la porte est en bois, la porte là-bas, on la touchera } \\
\text { tout à l'heure en sortant, mais la sculpture n'est } \\
\text { pas en bois }\end{array}$ \\
\hline M & $\begin{array}{l}\text { et puis elle est blanche, pas comme le bois c'est } \\
\text { marron }\end{array}$ \\
\hline une contemplation de quelques secondes)
\end{tabular}

Nous nous demandons à ce stade comment inscrire la compétence "choix du lexique " dans l'outil de formation: même avec les enfants très jeunes, les enseignants et les médiateurs professionnels apportent des éléments de savoir sous une forme explicite, par 
exemple sous la forme de termes techniques. La question renvoie très largement au niveau de langue auquel les élèves sont exposés. Il y a là des choix importants, si on considère que ce qui est en perspective, ce n'est pas moins que la production des inégalités culturelles par l'école (Bautier \& Rayou, 2009 ; Joigneaux, 2009). Jusqu'où trop d'adaptation au niveau supposé des élèves, à leur «manque de vocabulaire », contribue justement à renforcer les inégalités culturelles? Pour mémoire, on observera que ces interrogations sont celles du travail du médiateur professionnel: comment ajuster au mieux l'apport d'information à la demande d'un visiteur donné, et à sa capacité d'attention et de compréhension?

\section{Faire parler plusieurs langues et mettre en écho les formes multimodales d'existence des savoirs en jeu : formes verbales, formes matérielles, formes expérientielles}

Ce que fait apparaitre le travail du LéA Musécole sur la problématique de l'approche tactile de la sculpture, c'est en réalité la convergence des préoccupations d'acteurs qui peuvent apparaitre, au départ, comme évoluant dans des univers différents. Par exemple, nombre des questions que se posent les médiateurs ne sont pas autre chose que les questions des chercheurs que la problématique de l'approche tactile intéresse, comme l'ont montré les présentations et les débats lors du Fabre Lab. La problématique de l'approche tactile dans son lien avec l'approche visuelle intéresse la psychologie de la perception, et de l'art, mais c'est aussi une question centrale de l'éducation artistique, voire de processus d'apprentissage, selon les travaux des sciences cognitives: la multimodalité serait de nature à favoriser les apprentissages (Jewitt \& Kress, 2003), y compris en éducation artistique (Albers \& Sanders, 2010).

Encadré 2. Coopérer pour (s')apprendre à voir

Dans la Galerie tactile, autour de Statue équestre de Bartolomeo Colleoni, de J. M. Ramus $(1855$, h. 65), d'après A. del Verrocchio, une dizaine d'élèves se regroupent autour de la statue équestre en modèle réduit pour un échange guidé par l'enseignant. Une des élèves du groupe parle plus, mais les autres suivent son commentaire en allant toucher les parties qu'elle désigne et en intervenant. Les échanges portent sur des détails qui ont surpris les élèves. Ils ont rapidement reconnu un cavalier et son cheval, mais s'arrêtent sur l'arme qu'il porte, et aussi sur des détails très fins : les jambes, les bottes, des éléments en relief que la perception par le toucher a fait saillir ; le détail du casque, sa visière, et le couvre-nuque, sur lequel on revient à plusieurs reprises. Finalement, une comparaison va éclairer les explorateurs (nombreux acquiescements, reprise de la description) : le cavalier apparait équipé " comme les gendarmes en Algérie » : jambières, bouclier, et surtout casque. Est-on si loin, avec cette actualisation, de ce que le cavalier de Verrocchio pouvait signifier, la prestance un peu effrayante, la posture droite et assurée de l'homme en armes?

Il n'est pas sans intérêt d'observer que les concepteurs de l'exposition tactile ont prévu un premier espace d'accueil qui propose des expériences de toucher en aveugle, dans des boites à toucher : c'est un dispositif bien connu des classes maternelles, de même que les apprentissages tactiles des matières, tissus, pierres, métaux, papiers... Il faut souligner 
que le lien entre verbal, visuel et tactile est direct et permanent : il s'agit tout autant de sentir avec les mains (ou avec les pieds nus, le corps tout entier) que de ressaisir ces sensations par du langage immédiat ou de rappel, pour nommer les matériaux, les matières, les surfaces, les sensations, aussi précisément que possible. Ce lexique du toucher est explicitement listé dans les dossiers pédagogiques. Réciproquement, il sert à préparer les approches techniques de l'objet sculpté et du geste du sculpteur.

Une autre section de l'exposition illustre d'autres liens possibles: la galerie tactile présente dans un de ses tableaux certains des outils (le marteau, le marteau à dent, le rabot, la masse, le fil à plomb, la règle, l'équerre...) et des matériaux du sculpteur (bois, pierre, granit, marbre, argile, terre, plâtre, fer, bronze, ouvrant sur des actions spécifiques : la taille, le modelage, le moulage, la fonte); mais ils sont fixés sur la tablette pour des raisons évidentes de sécurité. Il manque ici bien sûr un atelier de manipulation directe, dont on comprend qu'il ne puisse prendre place dans une salle de musée. Mais les élèves ont cette chance qu'eux pourront avoir appliqué ces mêmes outils à ces mêmes matériaux; dans la classe les élèves pourront avoir, aussi longtemps que nécessaire, l'expérience directe de reproduire les mêmes gestes du modelage, de la découpe, de l'accumulation, de l'assemblage, du moulage...; gestes qui vont donner leur sens à une entrée dans la sculpture via un langage corporel, toujours doublé de ses verbalisations. Là, en quelque sorte, le musée a «besoin » de ces ateliers externes pour prolonger ses propres propositions ${ }^{7}$ ou pour les préparer.

Il en va de même avec la vidéo installée dans la galerie tactile : l'écran est installé dans un lieu de passage, la vidéo est assez longue: les visiteurs s'y attardent rarement. Typiquement, c'est dans la classe qu'on aura le temps de la regarder, mais aussi de la commenter. Ainsi, le dispositif muséal inscrit dans sa scénographie des références directes à des types d'activité qui ne peuvent prendre tout leur développement que dans un autre temps et un autre espace: le lien avec le temps scolaire s'y inscrit très directement : pour profiter pleinement de ces espaces de transition et de rappel entre les deux mondes, le travail de médiation-action a besoin du temps long des séances de préparation et de prolongement pour prendre tout son sens. Le lien espace muséal-espace scolaire est bien plus serré, en réalité, que les acteurs n'en ont conscience.

\section{Dire pour faire-voir ; dire pour faire-agir}

Les observations montrent aussi qu'il faut se garder de limiter le langage de l'incitation à la seule modalité verbale. Une partie des consignes de l'enseignant passe aussi par le langage corporel, paraverbal, mimique, gestuelle, posture.

Quand Manon, devant L'escrimeur de la salle Richter, double ses questions sur le personnage, en esquissant un mime, elle déclenche toute une série d'esquisses de mimes chez les élèves, qui se prennent au jeu et se "parlent » mutuellement en reproduisant la posture sculptée. Manon sait arrêter le jeu verbal-postural au moment où l'on voit que se désynchronisent les jeux de regards, les verbalisations, et les mouvements du corps qui « tâtonnent " pour retrouver le mouvement sculpté.

31 Ces procédés d'approche mimétique sont désormais bien connus parmi les techniques de médiation-métier ${ }^{8}$, et confirmés par ce que l'on sait désormais des processus perceptifs (liens entre perception visuelle, empathie et motricité). On peut aussi ici observer que le langage corporel prend le relais de ce qui serait très difficile à décrire, à savoir que la sculpture ici saisit ce que les danseurs appellent un état de corps, qui est plus qu'une 
simple position dans l'espace, mais une signification beaucoup plus globale et complexe, un bouger-sentir-exprimer que la verbalisation peine à traduire. Le langage corporel prend le relais du langage verbal, mais sans perte ni réduction.

D’une manière plus générale, la compétence professionnelle ici évoquée se rapproche d'un savoir faire tout à fait typique des enseignements artistiques : l'enseignant comme le médiateur sont ceux qui savent parler pour faire faire. Cela va tellement de soi qu'on oublie que ce travail d'incitation est à la fois très diversifié, très précis et qu'il est nourri d'une réflexion précise sur le sens des actions sollicitées (Cotner, 2011; Rickenmann, 2002). Qu'est-ce qui est précisément demandé aux élèves ? Les échanges font apparaitre que les enseignants comme les médiateurs utilisent un jeu de questions pré-définies, qu'ils peuvent faire varier et reformuler, et qu'ils présentent dans un ordre qui peut varier. Cette simple liste de consignes est un outil important pour la formation, si on n'oublie pas qu'elle est doublée par un autre type de savoir-faire, celui qui permet de ne pas l'appliquer de manière mécanique.

Une partie de l'outil en cours d'élaboration cherche à lister les principales catégories d'incitation en situation de réception, les « consignes-types » dont le médiateur dispose et qu'il ajuste à ses publics. Le recensement de ces formes récurrentes relève de la description des gestes de métier. La catégorie générale « faire parler » est trop imprécise pour rendre compte du jeu de conduites et des "genres verbaux » caractérisables de manière plus fine, en termes d'actes de langage ou de microgenres: les élèves n'apprennent pas seulement à " parler ", ils apprennent précisément à nommer, à poser des questions choisies dans un certain répertoire, à exprimer une émotion, à rappeler une expérience, à développer une fiction, etc.

Sur le plan didactique, ces règles implicites de la prise de parole constituent une partie du contrat, ce qui est compris comme attendu par les acteurs. Par exemple, que signifie exactement la question «que voyez-vous? » La question porte-t-elle sur l'objet matériel, sa dimension, sa matière? Porte-t-elle sur ce que l'on «imagine voir» (on a le droit d'inventer) ou sur ce que l'on « doit voir » (il faut chercher alors quelle est l'intention, la proposition, ou les interprétations qui ont été données) ? On mesure aussi que chacun des microgenres appelle des compétences dont certaines d'entre elles sont spécifiques du domaine : un exemple peut être donné par les «questionnaires-types » qui sont fournis dans les guides pédagogiques remis aux enseignants, ou remis aux étudiants pour préparer une lecture d'œuvre en histoire de l'art (Dumortier, 2004 ; Ricker, 2011).

\section{L'art d'écouter ; le silence comme art de faire}

35 Un autre élément clé est en lien avec la problématique des langages et des signes. Les analyses croisées font apparaitre que les intervenants se montrent capables de commenter de manière très fine les conduites visibles de leurs élèves, et « lisent » à la fois leurs paroles, leurs regards, leur gestuelle, leur corps. C'est d'autant plus nécessaire que ces élèves très jeunes sont justement là pour apprendre les conduites discursives qui se tiennent dans ces situations, mais aussi que les en-jeux d'apprentissage ne sont pas seulement de l'ordre du verbal : se rendre sensible, être attentif, se concentrer, prendre le temps d'imaginer, etc. Les professionnels du LéA se montrent capables de lire, chez des élèves très jeunes et souvent petits parleurs, des signes pour eux non ambigus de leur attention ou de leur mise au travail: les élèves bougent/s'immobilisent, grimacent, désignent, fixent leur regard, inclinent la tête, miment, désignent... Une des difficultés 
rappelées est d'orchestrer toutes ces partitions dès lors que les groupes sont plus nombreux, et de ne pas presser le pas de ce dialogue partiellement muet. Comment développer en formation cette capacité d'écoute? L'outil en construction propose des vidéos à analyser sous cet angle, en s'intéressant paradoxalement aux apparents « silences sémiotiques » dans les situations.

\section{Faire parler d'autres langues}

Un des contenus de formation qui émerge comme crucial dans les analyses de situation est l'exploration de dispositifs (modestement) plurisémiotiques/multimodaux. La verbalisation est rarement isolée ; elle s'articule avec d'autres usages de signes, comme on l'a vu pour la sphère corporelle/motrice. Savoir jouer de ces entrelacements est un art de faire professionnel. Prenons un exemple : faire dessiner les élèves [avant-pendant-après] la visite. La plupart des séances observées comportent des moments où les élèves sont invités à dessiner, soit devant pendant la visite, soit immédiatement après, soit plus tard. Certains outils préparés par le service des publics incluent des supports à dessiner.

En formation, il s'agit de comprendre ce qu'enseignent ces tâches graphiques, qu'elles ne sont pas marginales ou récréatives, mais qu'elles jouent aussi un rôle instrumental majeur pour construire par exemple capacité d'attention et de mémorisation, voir traduction d'un ressenti. Le dessin n'est pas seulement la traduction d'une perception visuelle qui se serait construite indépendamment d'elle, c'est le moyen par lequel elle s'intensifie, se densifie : comme le commentaire verbal peut étayer le parcours visuel ou haptique, la tâche de dessiner sollicite une forme de perception affinée et intensifiée (Wollheim, 1988). La tâche de dessiner est un catalyseur de l'attention, bien documentée en psychologie du dessin et en éducation artistique (Theron et al., 2011) ${ }^{9}$. C'est un langage pour l'expérience, et pas seulement un moyen de l'exprimer. C'est donc bien une compétence professionnelle que de savoir regarder les travaux des élèves comme des œuvres en écho, et pas seulement les stocker négligemment en fin de séquence. On pourrait ajouter comme une autre compétence professionnelle, la conduite même de la séquence de dessin, comment elle est organisée et guidée, ce qui est en soi un objet de réflexion.

En effet, la fonction instrumentale du dessin ne s'arrête pas à la modalité visuelle : comme dans les pratiques de référence, les traces graphiques sont mises en commun, discutées, confrontées aux œuvres originales. Elles alimentent en retour d'autres conduites verbales et d'autres retours vers les œuvres. Ce sont pleinement des instruments pour la médiation, l'éducation à l'attention à l'œuvre (Heath \& Wolf, 2005).

Encadré 3. D’autres « langages » sollicités, par exemple le dessin

Dans les 40 minutes prévues pour la visite de la galerie tactile, Philippe insère quelques minutes où les élèves, après avoir parcouru la salle les yeux bandés, sont invités à dessiner l'une des œuvres qu'ils ont touchées, à leur choix. C'est cette préparation par le dessin qui explique en grande partie la richesse des échanges autour de l'œuvre, où se mêlent à la fois le verbal (nommer les éléments plastiques, les resituer dans des contextes), le graphique (la consigne est : « vous vous souvenez de ce que vous venez de dessiner ? »), et le tactile : les élèves, même silencieux, touchent très longuement les détails, parfois très fins, qui sont évoqués et questionnés (voir l'encadré, § 2) 
On pourrait ici évoquer l'usage d'autres langages comme outil et mémoire de l'expérience : la photographie par exemple.

Encadré 4. D'autres outils sollicités : faire de la photographie un langage pour revenir à l'expérience

Pour mémoire : Philippe utilise systématiquement des tablettes pour photographier, dans sa classe, les productions des élèves. Il ne s'agit pas de garder une trace, mais de doubler l'objet d'une image qui servira dans des séances de rappel ; et aussi de travailler sur la photographie comme langage : choix du cadre, de l'angle, etc.

\section{La gestion empathique de l'attention et le partage du sensible}

Un des arts de faire paradoxaux de la médiation-action est l'art de faire voir, de guider l'appréhension visuelle, sensible. Paradoxal, car il semble à première vue que la perception relève d'une expérience radicalement subjective. C'est pourtant une grande partie du travail que d'étayer le parcours attentionnel, d'une part, mais aussi le développement des conduites d'appréhension « sensible »; il y a là une problématique qui intéresse autant la sociologie des dispositions (comment se construit l'hexis corporel [Durand, 2016]) que la psychologie de l'expérience esthésique/esthétique (Schaeffer, 2015).

Un autre des savoir-faire que nous cherchons à identifier pour le présenter en formation relève d'une question fondamentale pour l'éducation esthétique : comment apprend-on à être sensible, au sens d'être capable des formes d'expérience qui sont supposées être spécifiques des situations types en ÉAC, quand on parle de "rencontre avec des œuvres »? J.-M. Schaeffer (ibid.) caractérise la dimension esthétique de l'expérience comme sollicitant une forme particulière d'attention, d'intellection et d'émotion, qui, à partir de bases biologiques partagées, relèvent d'une éducation spécifique à ce qu'on appellera la sensibilité esthétique. Mais dans le processus même de cette éducation, saisie à une échelle fine, comment ça se passe? Qu'est-ce qui s'apprend devant l'œuvre? Une partie de la réponse est donnée par les phénomènes d'empathie (Gefen \& Vouilloux, 2013). Une partie des responsabilités du médiateur est là, dans la capacité à intensifier et maitriser, d'une certaine manière, la gestion de phénomènes conjoints : ce qu'on pourrait appeler une appréciation conjointe, un partage de l'expérience sensible dans sa dimension perceptive (la gestion de l'attention conjointe, par exemple "regarder ", « toucher » ensemble), émotive (partager ou confronter des ressentis, partager des états de corps), cognitive (nommer ces ressentis, les verbaliser : décrire, raconter, imaginer, interpréter...), axiologique (attribuer des valeurs différentielles) (White, 2013). Comment s'apprend justement ce qu'on appelle la sensibilité, soit non seulement la capacité à percevoir, mais aussi celle à réagir à l'œuvre?

Ici se joue peut-être le lien entre la dimension sociale et la dimension cognitive : les enjeux les plus profonds, les plus décisifs, relèvent des savoir être, des attitudes, des dispositions qui sous-tendent les apprentissages plus formels : l'observation précise des médiations permet de s'interroger sur des notions récurrentes dans les discours prescriptifs : "sensibilité », "curiosité », " capacité d'appréciation ", "éducation du gout ». Ces phénomènes ne sont pas livrés au hasard; l'art de faire du médiateur comme de l'enseignant est de densifier ces moments de partage du sensible, au plus près des corps en co-action. 
Nous en avons donné une illustration avec les deux situations où les incitations de l'enseignant accompagnent le parcours tactile (autour de La Frileuse, autour de la statue équestre).

\section{Conclusion} en laissant le plus possible la parole aux élèves, sans parler à leur place - tout en apportant de quoi parler? Il s'agit d'enchainer sur des énoncés parfois très brefs, un mot, une exclamation, un début de récit, une proposition énigmatique et lacunaire. On observe que les médiateurs-acteurs sont en particulier attentifs aux inventions verbales des élèves, qui sont des signes de leurs tentatives de formuler du difficile-à-dire. Mais le problème du professionnel est de prolonger et de solliciter des reformulations, d'enchainer sur des propositions pour développer des fils de conversation, de contrôler les digressions... 
47 On ne peut répondre à ces questions par des règles d'action qu'il suffirait d'appliquer pour réussir. Ce qui est décrit ici est précisément un savoir complexe, un savoir d'action, impossible à formaliser ou à réduire à quelques principes. C'est ici une compétence qui ne peut se construire que par une pratique réfléchie, sur des situations emblématiques, dont la densité rassemble ces questions jugées clés par les acteurs. C'est la visée de l'outil de formation que souhaite expérimenter le LéA :

- La liste des questions-types ne semble pas infiniment ouverte (voir ce qui est dit plus haut des « actes de langage » repérables); ce jeu de questions lui-même est un savoir, un élément de méthode. Elles sont la transposition des questions-clés des sciences de référence (« qu'est-ce qu'on voit? » : sémiologie ; « qui l'a fait et pourquoi ? : histoire de l'art; « que signifie... » : iconologie ; « à quoi ça fait penser ? » : histoire des réceptions ; etc.).

- Le lexique constitue sans doute un enjeu majeur, mais il pose une question délicate de réglage: à quel niveau de formulation doit-on se placer? Les dossiers pédagogiques proposent des listes de notions, une terminologie. Pour les enseignants, double problème : maitriser ce vocabulaire, faire le choix de ce qu'ils en reprennent à l'adresse des élèves. Le choix des enseignants du LéA est de faire le choix d'un lexique technique, doublé de traduction, mais présenté comme tel, sans attendre nécessairement que les élèves « soient au niveau ».

Cette question du réglage de la difficulté lexicale rejoint la tension entre approche sensible qui se passerait de mots à la limite, et approche réfléchie, qui solliciterait tout l'éventail des savoirs de référence : savoirs sur les œuvres, leur fabrication, leur histoire comme artefacts, leurs interprétations. Ces savoirs, qui ont une forme déclarative (ce sont des mots, des textes, des récits, des descriptions, des explications...) ne sont pas séparables de savoir-faire de tous ordres : poser des questions à une œuvre, par exemple. Pour autant, la frontière entre un lexique spécialisé et un lexique général est poreuse : l'exposition tactile ne sollicite pas moins que le lexique des matériaux, des sensations tactiles, etc.

Car le fil volontairement suivi dans cette présentation est la question du et des langage(s), langage(s) comme forme et matière du savoir, comme trace de l'activité de l'élève, comme outil très concret du travail de l'enseignant (des gestes professionnels langagiers [Bucheton \& Soulé, 2009]). La perspective applicative impose de dépasser la seule description pour s'imposer des mises en relief, toujours discutables, pour faire ressortir des priorités, des moments-clés où se manifestent des questions-clés et des savoir-faire-clés qu'on peut désigner comme des gestes professionnels langagiers (avec cette précision: bien souvent plurisémiotiques, entre modalités verbales, corporelles et visuelles). Ces objets sont au centre des dilemmes professionnels, et il ne s'agit pas d'en faire des préceptes ou des bonnes pratiques à simplement reproduire. Les poser, en débattre, les confronter aux choix individuels, une formation conçue comme un développement $\mathrm{du}$ pouvoir d'agir et non la transmission de formes prescrites.

Ce qui ici intéresse des chercheurs venus des sciences du langage est que ce point de vue s'inscrit dans un modèle des apprentissages qui les décrit comme portés par les négociations sémiotiques, non pas simplement transmis, mais co-construits et négociés (Bernié, Jaubert, \& Rebière, 2003). Ces négociations ne sont pas pour autant infiniment diversifiées, elles s'inscrivent dans des formes, des genres, qui définissent un savoir-faire professionnel par des régularités. Et pour autant, il ne s'agit pas seulement de techniques au sens étroit, mais d'arts de faire, dont l'histoire des arts du langage garde la trace depuis les problématiques de l'ekphrasis (Vouilloux, 2005) jusqu'à celle des écrits sur l'art 
(Vaugeois, 2005) et des formes discursives de la critique d'art (Leeman, 2010) ou de l'histoire de l'art (Zerner, 1997). Cela peut-être peut se résumer à une règle : prendre très au sérieux le travail des élèves devant les œuvres, et sa dimension langagière.

\section{BIBLIOGRAPHIE}

AHR, S. \& JOOLE, P. (éds) (2013). Carnet/journal de lecture/lecteur. Quels usages, pour quels enjeux, de l'école à l'université? Namur : Presses universitaires de Namur.

ALBERS, P., \& SANDERS, J. (éds). (2010). Literacies, the arts and multimodality. Urbana, Ill : National Council of Teachers of English.

ALIN, C. (2010). La geste formation. Gestes professionnels et analyses des pratiques. Paris : L'Harmattan. ARBOUET, F. et al. (2014). Dossier pédagogique d'accompagnement à la visite en autonomie de l'exposition Claude Viallat, une rétrospective (juin à nov. 2014) (Dossier réalisé dans le cadre de la convention de partenariat entre l'ESPÉ/FDE/UM2 et le Musée Fabre). Montpellier : Musée Fabre. En ligne : http://museefabre.montpellier3m.fr/content/download/9029/73577/file/ Dossier_pédagogique_visiteautonomie_Viallat.pdf.

BAMFORD, A. (2008). «Mesurer l'impact : Recherche(s) en éducation artistique et culturelle ». In : Rouhète, M. (éd.), Évaluer les effets de l'éducation artistique et culturelle. Paris : La Documentation française/Centre Pompidou, p. 21-29.

BARTON, G. (2013). « The Arts and Literacy: What Does it Mean to be Arts Literate? » International Journal of Education \& the Arts 14, 18.

BAUTIER, É. \& RAYOU, P. (2009). Les inégalités d'apprentissage. Programmes, pratiques et malentendus scolaires. Paris : Presses universitaires de France.

BEAUDE, P.-M., PETITJEAN, A., \& PRIVAT, J.-M. (éds) (1996). La scolarisation de la littérature de jeunesse. Actes du colloque de Metz, 1995. Metz : CRESEF.

BERNIÉ, J.-P., \& GOIGOUX, R. (éds) (2005). « Les gestes professionnels [dossier] ». La Lettre de l'AIRDF 36.

BERNIÉ, J.-P., JAUBERT, M., \& REBIÈRE, M. (éds). (2003). Construction des connaissances et langage dans les disciplines d'enseignement. Bordeaux : IUFM/Université Victor Segalen Bordeaux 2.

BIDARD, M.-D. et al. (2013). Signac, Point par point. Classes de Maternelles. Dossier pédagogique d'accompagnement à la visite en autonomie de l'exposition Signac, les couleurs de l'eau (juillet à octobre 2013) (Dossier réalisé en partenariat avec un groupe de formateurs de l'Académie de Montpellier, dans le cadre de la convention de partenariat Musée Fabre/IUFM de Montpellier). Montpellier : Musée Fabre. En ligne : http://museefabre.montpellier3m.fr/content/download/6812/49511/ file/Dossier accompagnement visite autonomie Signac.pdf.

BORDEAUX, M.-C., \& KERLAN, A. (2015). L'évaluation des « effets » de l'éducation artistique et culturelle. Étude méthodologique et épistémologique (Rapport d'étape). Ministère de la Culture. 
BUCHETON, D., \& SOULÉ, Y. (2009). «Les gestes professionnels et le jeu des postures de l'enseignant dans la classe : un multi-agenda de préoccupations enchâssées ». Éducation \& Didactique 3, 3, p. 29-48. En ligne : http://journals.openedition.org/educationdidactique/543.

CAUNE, J. (1999). Pour une éthique de la médiation. Le sens des pratiques culturelles. Grenoble : Presses universitaires de Grenoble.

CAUNE, J. (2017). La médiation culturelle. Expérience esthétique et construction du Vivre-ensemble. Grenoble : Presses universitaires de Grenoble.

CHABANNE, J.-C. (2013). « Histoire des arts et jeux de langage : comment parler de l'œuvre? » Le

Français aujourd'hui 3, 182, p. 55-66. En ligne : https://www.cairn.info/revue-le-francais-aujourdhui-2013-3-page-55.htm.

CHABANNE, J.-C. (2017). « Approches interdisciplinaires des enseignements culturels et artistiques dans le cadre de l'enseignement de l'histoire des arts : un modèle complexe, une didactique impossible ?» Tréma 45, p. 59-69. En ligne : http://journals.openedition.org/trema/3501.

CHABANNE, J.-C., MONOD-ANSALDI, R., \& LOISY, C. (2016). « Faire le lien entre la pratique et la recherche pour transformer l'école ? Le dispositif LéA de l'IFÉ comme laboratoire de l'innovation en recherche-intervention-formation. Analyse d'un cas particulier d'un LéA impliquant une ESPÉ ». In : Marin-Porta, B. \& Berger, D. (éds), Recherches en éducation, recherche sur la professionnalisation. Consensus et dissensus. Paris : Réseau national des ESPÉ, p. 284-300.

CHEVALLARD, Y. (1991) [1985]. La transposition didactique. Du savoir savant au savoir enseigné. Grenoble : La Pensée sauvage.

COTNER, T. L. (2011). «Speaking of Art, Listening to What Teachers Are Saying ». Art Education 64, 2, p. 12-17.

DIDI-HUBERMAN, G. (1990). Devant l'image. Question posée aux fins d'une histoire de l'art. Paris : Éd. de Minuit.

DUMORTIER, J.-L. (2004). « Dire une rencontre avec l'œuvre d'art : quelles connaissances pour une compétence ?» In : Cramer, E., Ricker, M.-E. \& Somville P. (éds), Enseigner l'histoire de l'art. Un art et une histoire tournés vers le futur. Liège : Éd. du 17 mars.

DURAND, P. (2016). « Hexis ». In : Glinoer, A. \& Saint-Amand, D. (éd.), Le lexique Socius. En ligne : http://ressources-socius.info/index.php/lexique/21-lexique/40-hexis.

ECO, U. (2006) [2003]. Dire presque la même chose. Expériences de traduction. Trad. de l'italien par M. Bouzaher. Paris : Grasset.

ENFERT, R. d' (2003). L'enseignement du dessin en France. Figure humaine et dessin géométrique (1750-1850). Paris : Belin.

FOUCAMBERT, J. et al. (1982). Lire, c'est vraiment simple... Quand c'est l'affaire de tous. Paris : OCDL. En ligne : https://www.lecture.org/ressources/BCD/lire_c_vraiment_simple.pdf.

FRANÇOIS, F. (1999). Le discours et ses entours. Essai sur l'interprétation. Paris : L'Harmattan.

GEFEN, A., \& vouilloux, B. (éds). (2013). Empathie et esthétique. Paris : Hermann.

HALTÉ, J.-F. (2008). « Le français entre rénovation et reconfiguration ». Pratiques 137-138, p. 23-38. En ligne : http://journals.openedition.org/pratiques/1150.

HEATH, S. B., \& WOLF, S. (2005). « Focus in creative learning: drawing on art for language development ». Literacy 39, 1, p. 38-45. 
HEINICH, N. (2009). Faire voir. L'art à l'épreuve de ses médiations. Bruxelles : Les Impressions nouvelles.

JEWITT, C. \& KRESS, G. (éds) (2003). Multimodal literacy. New York : P. Lang.

JOIGNEAUX, C. (2009). « La construction de l'inégalité scolaire dès l'école maternelle ». Revue française de pédagogie 169, p. 17-28. En ligne : http://journals.openedition.org/rfp/1301.

JORRO, A. (2006). « L'agir professionnel de l'enseignant ». Conférence au séminaire de recherche du Centre de Recherche sur la Formation. Paris : CNAM, 28 févr. En ligne : https://halshs.archivesouvertes.fr/halshs-00195900/document.

KASTRUP, V., \& SAMPAIO, E. (2012). « Le rôle de l'expérience esthétique tactile dans l'apprentissage des personnes handicapées visuelles dans les musées ». Savoirs 1, 28, p. 93-111. En ligne : https:// www.cairn.info/revue-savoirs-2012-1-page-93.htm.

LAMIZET, B. (1999). La médiation culturelle. Paris : L'Harmattan.

LEEMAN, R. (2010). Le Critique, l'art et l'histoire. De Michel Ragon à Jean Clair, 1959-1972. Rennes : Presses universitaires de Rennes.

LEMOINE, C., \& SIMIER, A. (2016). « La sculpture sur le bout des doigts. Retour sur l'élaboration d'une salle pédagogique et tactile au musée Bourdelle ». Les cahiers de l'École du Louvre 8. En ligne : http://journals.openedition.org/cel/341.

MAYZAUD, Y. (2005). « Historique et enjeu de la notion d'Erlebnis ». CENIPHE 2. En ligne : http:// ceniphe.free.fr/revue2_files/Y. mayzaud, Historique et enjeu de la notion d\%27Erlebnis.pdf.

MESMIN, P., \& BIDARD, M.-D. (2014). Parcours Maternelle en autonomie. Portraits-puzzles (Dossier pédagogique d'accompagnement à la visite en autonomie de l'exposition François-André Vincent (févr. à mai 2014)). Montpellier : Musée Fabre. En ligne : http://museefabre.montpellier3m.fr/ content/download/7260/55443/file/Visite en autonomie Maternelle Portrait-Puzzle VINCENT.pdf.

MINISTÈRE DE L'ÉDUCATION NATIONALE (MEN) (2008). Organisation de l'enseignement de l'histoire des arts (école, collège, lycée) (arrêté du 11-7-2008). Bulletin Officiel nº 32 du 28 août. Encart. En ligne : http://cache.media.education.gouv.fr/file/32/09/0/encart_33090.pdf.

MINISTÈRE DE L'ÉDUCATION NATIONALE (MEN) (2015a). Parcours d'éducation artistique et culturelle (arrêté du 1-7-2015). Bulletin Officiel du 8 juillet, (28). En ligne : http://www.education.gouv.fr/ pid25535/bulletin_officiel.html?cid_bo=91164.

MinistÈRe De l'ÉDucATion Nationale (MEN) (2015b). Programme d'enseignement de l'école maternelle (arrêté du 18-2-2015). Bulletin officiel spécial no 2 du 26 mars. En ligne : http:// www.education.gouv.fr/pid25535/bulletin_officiel.html?cid_bo=86940.

MiNiSTÈRE DE L'ÉDUCATION NATIONALE (MEN) (2015c). Programmes d'enseignement du cycle des apprentissages fondamentaux (cycle 2), du cycle de consolidation (cycle 3) et du cycle des approfondissements (cycle 4). Bulletin officiel spécial no 11 du 26 novembre. En ligne : http:// cache.media.education.gouv.fr/file/

MEN_SPE_11/67/3/2015_programmes_cycles234_4_12_ok_508673.pdf.

MiNiSTÈRE DE L'ÉDUCATION NATIONALE (MEN) \& MiNiSTÈRE DE LA CULTURE ET DE LA COMMUNICATION (MCC)

(2015). Éducation artistique et culturelle, éducation aux médias et à l'information. Malakoff, 15 février 2015 (Une feuille de route conjointe du ministère de l'Éducation nationale, de l'Enseignement supérieur et de la Recherche et du ministère de la Culture et de la 
Communication). Paris : MENESR/MCC. En ligne : http://cache.media.education.gouv.fr/file/02__fevrier/27/2/DP_Education_artistique_culturelle_bdef_391272.pdf.

MiNISTÈRE DE LA CULTURE ET DE LA COMMUNiCATION (MCC) (2017). Circulaire du 10 mai 2017 relative au développement d'une politique ambitieuse en matière d'éducation artistique et culturelle, dans tous les temps de la vie des enfants et des adolescents (NOR : MCCB1712769C). Paris : MCC. En ligne : http://circulaires.legifrance.gouv.fr/pdf/2017/05/cir_42213.pdf.

MÖRSCH, C., \& CHRUSCIEL, A. (éds) (2012). Time for Cultural Mediation. Zurich : Institute for Art Education of Zurich University of the Arts. En ligne : http://www.kultur-vermittlung.ch/zeitfuer-vermittlung/v1/?m=10\&m2=8\&lang=e.

Pratiques 147-148 (2010). «Interactions et corpus oraux ».

RICKENMANN, R. (2012). « La construction sociale de l'émotion esthétique : dimensions transactionnelles des rapports à l'objet culturel en classe ». In : Chabanne, J-C., Parayre, M. \& Villagordo, É. (éds), La rencontre avec l'œuvre. Éprouver, pratiquer, enseigner les arts et la culture. Paris : L'Harmattan, p. 317-336.

RICKER, M.-É. (2011). « Analyser les œuvres d'art au musée : quels moyens langagiers ? » Repères 43, p. 127-151. En ligne : http://journals.openedition.org/reperes/224.

ROUXEL, A. \& LANGLADE, G. (éds) (2004). Le sujet lecteur. Lecture subjective et enseignement de la littérature. Rennes : Presses universitaires de Rennes.

SANCHEZ, É., \& MONOD-ANSALDI, R. (2015). « Recherche collaborative orientée par la conception. Un paradigme méthodologique pour prendre en compte la complexité des situations d'enseignement-apprentissage ». Éducation \& Didactique 9, 2, p. 73-94.

SCHAEFFER, J.-M. (2015). L'expérience esthétique. Paris : Gallimard.

THERON, L. et al. (éds). (2011). Picturing research. Drawing as visual methodology. Rotterdam : Sense Publishers.

VAugEoIs, D. (éd.) (2005). « L'écrit sur l'art : un genre littéraire? » Figures de l'art 9.

vouilloux, B. (1997). Langages de l'art et relations transesthétiques. Paris : Éd. de l'Éclat.

vouilloux, B. (2005). « Texte et image ou verbal et visuel ?» In : Louvel, L. \& Scepi, H. (éds), Texte/ Image. Nouveaux problèmes. Rennes : Presses universitaires de Rennes, p. 17-31.

WEBB, R. (1999). « Ekphrasis Ancient and Modern: the Invention of a Genre ». Word \& Image 15, 1, p. 7-18.

WHITE, B. (éd.) (2013). Aesthetics, empathy and education. New York : P. Lang.

WINNER, E., GOLDSTEIN, T., \& VINCENT-LANCRIN, S. (2014). L'art pour l'art ? L'impact de l'éducation artistique, la recherche et l'innovation dans l'enseignement. Paris : Éd. OCDE.

Wollheim, R. (1988) [1965]. « Dessiner un objet ». In : Lories, D. (éd.), Philosophie analytique et esthétique. Paris : Klincksieck, p. 239-266.

ZERNER, H. (1997). Écrire l'histoire de l'art. Figures d'une discipline. Trad. de l'anglais par J. Bouniort. Paris : Gallimard. 


\section{NOTES}

1. L'équipe du LéA Musécole-Montpellier: Françoise Arbouet (enseignante-formatrice, école primaire Condorcet); Danielle Bertrand (enseignante-formatrice, école maternelle Virginia Woolf); Marie-Do Bidard (formatrice, doctorante); Jean-Charles Chabanne (enseignantchercheur); Manon Gaquerel (médiatrice, service des publics); Philippe Mesmin (enseignant formateur, école maternelle Virginia Woolf); Mary-Ève Penancier (docteure formatrice); Stéphanie Rieu (école maternelle Pauline Kergomard); Cécile Salvador (école maternelle Pauline Kergomard). Voir les productions du LéA sur le site du musée : Arbouet et al., 2014 ; Bidard et al., 2014 ; Mesmin et Bidard, 2014)

2. Voir également SENSEVY, G. (2011). «Vers une nouvelle alliance entre professeurs, éducateurs et chercheurs, entre recherche et formation?». En ligne: http://www.canal-u.tv/video/ ecole_normale_superieure_de_lyon/

vers_une_nouvelle_alliance_entre_professeurs_educateurs_et_chercheurs_entre_recherche_et_formation.10447 et SENSEVY, G. (2014). « La recherche dans les LéA, quelques éléments de réflexion après trois ans d'existence du dispositif». En ligne: https://www.canal-u.tv/video/ ecole_normale_superieure_de_lyon/

la_recherche_dans_les_lea_quelques_elements_de_reflexioin_apres_trois_ans_d_existence_du_dispositif.17303

3. Le Jacques Cour d'A.-A. Préault (1873, h. 245 cm) ; La Frileuse (1783, h. 145), L'été (1785, h. 155), Voltaire (1780-90, h. 145), de J.-A. Houdon; Tête de l'éloquence d'A. Bourdelle (1923, h. 49) ; L'enfant à l'oie, copie d'une statue du II ${ }^{\mathrm{e}}$ s. (h. 92); L'Amour au papillon d'A.-D. Chaudet (1817, h. 77) ; Statue équestre de Bartolomeo Colleoni de J. M. Ramus (1855, h. 65), d'après A. del Verrocchio; La Vierge et l'enfant entourés par quatre anges (dite aussi la Madone d'Auvillers) d'A. di Duccio (1469, bas-relief, h. 81) ; Jaguar dévorant un lièvre d'A. L. Barye (ap. 1850, h. 41) (I. SOBCZAK, 2016, Dossier pédagogique «L'art et la matière. Galerie de sculptures à toucher». Montpellier: Musée Fabre. En ligne : http://museefabre.montpellier3m.fr/content/download/11662/88994/file/

Art_et_Matiere_NOTICES_oeuvres_et_LEXIQUE.pdf, p. 19-20).

4. Nous utilisons l'expression «en-jeu » pour désigner toute forme de contenus d'apprentissage ou d'effets éducatifs que viserait une situation d'ÉAC, dans un sens volontairement plus ouvert et exploratoire que contenu (didactique), savoir, (objectif d') apprentissage, trop étroitement liés à une définition scolaire des visées de l'ÉAC. Le terme élargit encore la notion de jeu que G. Sensevy reprend de celle de L. Wittgenstein.

5. Fabre Lab : de la main à l'esprit, toucher pour mieux connaitre en 2017. Sur une série de trois séminaires organisés autour de l'exposition «L'Art et la matière », en partenariat avec la licence sciences du langage, parcours communication, médias et médiation numérique, de l'Université Paul-Valéry Montpellier 3, des chercheurs, des conservateurs, des médiateurs, des psychologues, et des professionnels du langage se sont réunis pour interroger le sens du toucher dans le rapport à l'art et la représentation du monde. (T. Martinez, «Exposition L'Art et la matière au musée Fabre ", 22/03/2017. Reportages. Montpellier. Radio Campus Montpellier. En ligne : http:// www.radiocampusmontpellier. $\mathrm{fr} / \mathrm{p}=3806$.)

6. R. Gayrard, Enfant jouant avec une levrette, XIX ${ }^{\mathrm{e}}$ siècle, Marbre.

7. Noter que le musée propose de manière permanente un atelier de pratique animé par des plasticiens. Le lien entre voir et faire est ici intégré au dispositif muséal, mais toujours à la suite d'une visite, et manque ici le bouclage entre les deux dimensions et son inscription dans le temps.

8. Voir par exemple l'atelier Mouvent sculpté-Mouvement dansé que proposait le musée du Louvre ( http://www.louvre.fr/ateliers/mouvement-sculpte-mouvement-danse-0) ; ou encore les ateliers 
Mimer la sculpture (http://www.louvre.fr/ateliers/mimer-la-sculpture-enfants-). Certains jeux de médiation proposent une vraie construction fictionnelle avec un jeu de pantomime, moitié ludique, moitié savant: Museum Hack (http://museumtwo.blogspot.fr/2014/12/how-museumhack-transforms-museum-tours.html).

9. Et en histoire de l'éducation : R. d'Enfert (2003).

\section{RÉSUMÉS}

Cet article présente l'état actuel des réflexions du LéA Musécole, un groupe de rechercheintervention enregistré dans le réseau LéA (un réseau géré par l'Institut français d'éducation reliant plus de 30 groupes de recherche-intervention). Le Musécole travaille sur la conception de ressources de formation numérique visant à développer la capacité de préparer, d'échafauder et d'enrichir les situations dans lesquelles les étudiants rencontrent des œuvres d'art. L'objectif est de les intégrer pleinement dans une continuité revendiquée entre le monde de l'école et les mondes de l'art, par opposition à certaines options éducatives qui conduisent à un temps, un lieu et un contenu isolés pour l'éducation artistique et culturelle. LéA Musécole vise à souligner et à favoriser la contribution indispensable à l'apprentissage scolaire de ce qui est en jeu dans les lieux de l'art. Mais il veut éviter toute instrumentation : ces expériences esthétiques nourrissent des opportunités stimulantes pour développer des «compétences culturelles » et des compétences multidisciplinaires avec un haut degré de demande, pourvu que leur spécificité soit pleinement respectée.

This article presents the current state of reflections of the LéA Musécole, a research-intervention group registered in the LéA network (a network managed by the French Institute of education linking more than 30 research-intervention groups). The LéA Musécole is working on the design of digital training resources aiming at developing the ability to prepare, scaffold and enrich situations in which students are encountering works of art. The aim is to fully integrate them in a continuity claimed between the world of school and the worlds of art, as opposed to certain educational options that lead to secluded time, place and content for artistic and cultural education. LéA Musécole aims to highlight and foster the indispensable contribution to school learning of what is at stake in the art places. But it wants to avoid any instrumentalization: those aesthetic experiences nourish stimulating opportunities to develop "cultural skills" and multidisciplinary skills with a high degree of demand - provided their specificity be fully respected.

\section{INDEX}

Mots-clés : éducation artistique et culturelle, arts et langue comme outil, éducation artistique et multilittéracie, approche interdisciplinaire de l'éducation artistique

Keywords : arts and cultural education, Arts and language as tool, Arts education and multiliteracy, Interdisciplinary approach of art education 


\section{AUTEURS}

\section{FRANÇOISE ARBOUET}

ESPÉ Languedoc-Roussillon, F-34000, France

DANIELLE BERTRAND

ESPÉ Languedoc-Roussillon, F-34000, France

\section{MARIE-DO BIDARD}

ESPÉ Languedoc-Roussillon, F-34000, France

JEAN-CHARLES CHABANNE

ENS de Lyon, ECP, EA 4571, F-69007, France

MANON GAQUEREL

Musée Fabre, Montpellier Méditerranée Métropole, F-34000, France

\section{PHILIPPE MESMIN}

ESPÉ Languedoc-Roussillon, F-34000, France

\section{MARY-ÈVE PENANCIER}

Université de Montpellier, ESPÉ Languedoc-Roussillon, LIRDEF, EA 3749, F-34000, France

STÉPHANIE RIEU

ESPÉ Languedoc-Roussillon, F-34000, France

CÉCILE SALVADOR

ESPÉ Languedoc-Roussillon, F-34000, France 